\title{
The promise of chalcogenides
}

\author{
Chalcogenide glasses are attracting significant attention thanks to their mid-infrared transparency and highly \\ nonlinear properties. Nadya Anscombe talks to Dan Hewak from the University of Southampton in the UK.
}

What are chalcogenide glasses and how are they different to silica?

Chalcogenides are compounds formed primarily from chalcogen elements such as sulphur, selenium or tellurium, and are very different from silica glass. Chalcogenides come in a variety of colours that depend on their chemical constituents, ranging from partially transparent to completely opaque. In contrast with silica, which is predominantly a passive material, chalcogenides exhibit active properties and are highly nonlinear. Chalcogenides are also photosensitive, which, although a potential disadvantage for some applications, is a significant advantage for writing structures into fibres. In addition, they are glassy semiconductors, which gives them interesting electrical properties.

\section{What are the potential applications?} Chalcogenide glasses in the form of thin films are already used in optical data storage and in the manufacturing of highly efficient solar cells. Chalcogenide fibres can be used for the transmission of light at wavelengths beyond those possible with silica. Shortly after the invention of the laser, chalcogenide fibres were studied as waveguides for delivering $\mathrm{CO}_{2}$ laser light at wavelengths of $10 \mu \mathrm{m}$. As new lasers were discovered, various chalcogenide compositions were developed to address individual wavelengths. Adding tellurium to the glass tends to increase transmission at infrared wavelengths, whereas sulphur aids transmission at visible wavelengths. Chalcogenides are also useful for medical applications such as laser surgery, which requires a wavelength of around $3 \mu \mathrm{m}$. Gallium lanthanum sulphide fibres transmit well at this wavelength, and their non-toxic components and high melting temperature suit minimally invasive surgery. At similar wavelengths, chalcogenide fibres have several atmospheric transmission windows for applications in spectroscopy and sensing.

\section{How are chalcogenide fibres made?} Like silica fibres, chalcogenide fibres can be drawn from a preform. However, unlike silica, the drawing temperature of a chalcogenide is close to its crystallization temperature. Crystallization weakens a fibre and alters its optical properties. The



Hewak: "We urgently need to find a reliable and reproducible way of making high-purity chalcogenide glasses, and I believe chemical vapour deposition could be the answer."

crucible drawing process is an alternative technique that can help prevent the formation of crystals. However, the main challenge is not drawing the fibre, but rather manufacturing a glass with high purity. The techniques we use today to manufacture novel glasses are the same as those used hundreds of years ago, but they are not suitable for making chalcogenide glasses because of the high purity levels required. Glass purity is of the utmost importance for both the scientific study and practical application of chalcogenide glasses. Varying levels of trace impurities, even at levels of a few parts per million, can alter the spectroscopic behaviour of a chalcogenide glass. In optical components such as fibres, impurities not only contribute to the optical loss through absorption and scattering but also serve as nucleation sites for crystallization. We urgently need to find a reliable and reproducible way of making high-purity chalcogenide glasses, and I believe chemical vapour deposition could be the answer. This process is capable of producing highpurity glasses at low cost because it relies on liquid-metal chloride precursors and operates at atmospheric pressure. However, this technique has a slow growth rate and the required precursors are often dirty and expensive.
What's been holding the area back? The chemistry of making chalcogenides is simple, but the engineering is complex. Theoretically, chalcogenide fibres have all the right properties to enable many exciting applications, but making fibres that match theoretical parameters is another matter. There was a time when fluoride fibres were tipped to be the next big thing; in theory, they could out-perform silica fibres and provide trans-Atlantic spans without the need for repeaters. There was a flurry of research activity, but no one managed to realize a fibre that could match the required performance characteristics, and the technology soon disappeared for telecommunications applications. If we stick to conventional methods of manufacturing chalcogenide fibres, we will see history repeat itself. I believe it is possible to make chalcogenide fibres with the optical parameters that we need, but only if we manage to develop new glass-melting technology and reproducible, reliable fabrication techniques.

Are there many start-ups in this area? Several research groups around the world are developing the technology required to make chalcogenide fibres, and a handful of companies have already started up in this field. One example is the Canadian company CorActive, which offers both sulphide glass (wavelengths of 2-6 $\mu \mathrm{m}$ ) and selenide glass (wavelengths of 2-9 $\mu \mathrm{m}$ ). The company uses state-of-the-art technology developed at the Naval Research Laboratory in the USA. The processes they use are repeatable and reliable, but also time-consuming and expensive. The chalcogenide fibre market therefore remains small and the fibres are expensive. This cost is predominantly due to the expense of the raw materials and manufacturing processes. As the applications of chalcogenide fibres become more widespread, the balance between cost and manufacturing quantity will shift. It takes only a little more effort to draw $1 \mathrm{~km}$ of fibre than $10 \mathrm{~m}$ - with increased demand the price per metre of chalcogenide fibre is certain to fall.

\section{NADYA ANSCOMBE}

Nadya Anscombe is a freelance journalist based in the United Kingdom. 